\title{
SCEAF IN DEN NORDISCHEN GENEALOGIEN.
}

In den nordischen fassungen der geschlechtsreihe Scéaf Wóden im Langfeðgatal (Langebek 1,2), in der Suorra Edda (AM. 2, 252 verglichen mit 1, 22 f.), der Sverrissaga (Flat. 2, 533) und in Frá Fornjóti (Flat. 1, 27) sind die ags. namensformen im allgemeinen gut bewahrt, böchstens etwas norrönisiert. Nur der name Scéaf erscheint tiberall in einer verderbten zweisilbigen form, die mit se oder ce anlautet: Seskef uel Sescef Langf., Seseph Fl. 1, Sesep Fl. 2, Sefsmeg SE. U, Cespheth W, Cesphete H. Diese entstellung des namens fuhrt mit sicherheit auf die ags. quelle aus welcher alle nordischen versionen unserer genealogie geflossen sind. Es ist dies die westsächsische genealogie in der bei Wright und Halliwell, Reliquiae antiquae 2,171 ff. aus dem codex Cotton. Tiberius $B$. $V$ veröffentlichten fassung, die ich mit $H$ bezeichnen will, und die in mebreren punkten von der uberlieferung der genealogie in der Sachsenchronik (zum jahre 855) abweicht. Das nähere ergibt folgende tabellarischo ubersicht:

\begin{tabular}{|c|c|c|c|c|}
\hline Ags. genealogien & Langf. & SE. & Fl. 1. & Fl. 2. \\
\hline Woden Frealafinz & Voden & Voddena & Voden & Odins \\
\hline af' Finninz & Frealaf & Frialafr b & Frialafr & Frialafs \\
\hline Godulfin $^{8}$ & Finn & Finnrc & Finn & Finz \\
\hline Potinz & Goðulfi & Guðolfr & Godolfr & Godo \\
\hline
\end{tabular}

1 Woden Fripowaldinz. Fripuwald Freawininz. Frealaf Fripuwulfinz. Fripuwulf $A$ 2 Finn $B H$, Fin die üibrigen 3 so $C D H$, Godwulfing $A B \quad 4$ so $B C D E$, Godwulf $A$ 5 so $\not H$, Gatinz $D$, Geatinz die übrigen

a Voðen $W$, Vópinn $R H$ b Friallaf $W$, Friallaff $H$, Fiarllaf $R$ c fehlt $R$ 


\begin{tabular}{|c|c|c|c|c|}
\hline Ags. genealogien & Langf. & SE. & Fl. 1. & Fl. 2. \\
\hline Eat ${ }^{1}$ Tætwainz & Eat & Jat & - & - \\
\hline 'I'ætwa Beawinz & - & - & - & - \\
\hline Beaw Scealdwazinz ${ }^{2}$ & Beaf & Biafa & Beaf & Biars \\
\hline Scealwa ${ }^{3}$ Heremodinz & Scealdna & Skialldun b & Skialldin & Skiallda \\
\hline Heremod Itermannin $z^{4}$ & ${ }^{4}$ Heremotr & Eremojc & Heremoth & Hermods \\
\hline Iterman $^{5}$ Haðrainz ${ }^{6}$ & Itermann & Itrman & 'Trinaan & 'Trinams \\
\hline $\begin{array}{l}\text { Hadra }[\text { Hwalainz } \\
\text { Hwala }]^{7} \text { Bedwizinz }\end{array}$ & Athra & Atrad & Atra & Atraas \\
\hline $\begin{array}{l}\text { Hwala }]^{\circ} \text { Bedwizing } \\
\text { Bedwiz Seeafinz }\end{array}$ & Beðvig & Beðvig $\theta$ & Beduigg & $\overline{\text { Beduigs }}$ \\
\hline $\begin{array}{l}\text { Se Scef wæs Noes } \\
\text { sunu etc. }{ }^{10}\end{array}$ & $\begin{array}{l}\text { Seskef uel } \\
\text { Sescef }\end{array}$ & Sefsmeg $f$ & Seseph & Seseps \\
\hline
\end{tabular}

1 so $H$, Geatt $C$, Geat die übrigen 2 so $H$, Scealdwainz $C D$, Sceldwainz $A$, Sceldweainz $B$ s so $H$, Scealdwa $C$, Scealdhwa $D$, Sceldwea $A$, Scyldwa $B \quad 4$ so $H$, -moninz die übrigen 5 so $H$, -mon die übrigen 6 so $B C H$, Hadrahinz $D$, Hrawrainz $A G$, das übrige fehlt $A G$ 7 Hwalainz Hwala $B C$, bloss Hwala $D$, fehlt $H \quad 8$ Beowunz $D 9$ Beowi $D 10$ Sceafinz. id est filius Noé $B C D$.

a Biaff $H$; der rest fehlt $R$ b Skialdunn $W$, Scjalldr $H$ c Heremóð $W$, Módar $H$ d Athra $W H$ e Ledinz $H$ f Cespheth $W$, Cesphete $H$.

Hier fällt zunächst der enge anschluss der nordischen uberlieferung an $\mathrm{H}$ in orthographicis ins auge. Nur $\mathrm{H}$ hat, dem nord. Eat, Jat entsprechend, die spätags. form Eat fur Geat (Ags. gr. \$212, anm. 2). Auch Iterman mit a kennt nur $\mathrm{H}$. Die form Godulf statt Godwulf teilt $\mathrm{H}$ zwar mit dem hss. BCD der Sachsenchronik, aber diese entfernen sich wider durch den einschub des Hwala vor Bedwiz vom nordischen. Vor allem aber steht $\mathrm{H}$ darin ganz allein, dass es statt des schlusses der reihe Bedwiz Sceafinz. id est filius Noe wie ihn BCD zeigen (in AG fehlt er ganz) den rein ags. text Bedwiz Sceafinz. se Scef waes Noes sunu u. s. w. bietet, wider mit der spätags. form Scéf statt Scéaf (Ags. gr. §102). Dieses se Scéf aber ist doch sicherlich die quelle des im Langfeðgatal getreulich erhaltenen Seskef uel Sescef, das dann in den ubrigen nordischen quellen weiter entstellt wurde. Derjenige welcher den ags. text zunächst excerpierte und in die nordische genealogie einstellte, liess, wie man sieht, die ags. patronymica auf -inz uberall aus, ubersah dabei das richtige Scéafinz nach Bedwiz und setzte blindlings das nun folgende se scef seiner vorlage als namen 
in seinen text! Eine drastischere illustration für die mechanische art der interpolation der ags. reihe in die nordische genealogie hätte sich wol kaum erwarten lassen. Man darf hiernach sicher behaupten, dass von einer auch nur einigermassen volkstimlichen tradition nicht die rede sein kann, welche etwa die ags. namen in die nordischen quellen gebracht hätte; vielmehr handelt es sich in erster instanz um eine rein gelehrte manipulation eines einzelnen, dem dann später andere nachschrieben.

Im einem punkte muss freilich die vorlage noch correcter gewesen sein als unser $\mathrm{H}$, bei dem namen Scealdwa, dessen $d$ in $\mathrm{H}$ fehlt, wenn der abdruck richtig ist. Aber gerade auch bei diesem namen zeigt sich wider wie mechanisch man bei der herübernahme verfuhr. Das Scealdwa der ags. quelle wird der nordische compilator zunächst wol durch Scealdua widergegeben haben (vgl. Beðuig, Beduigg etc. = ags. Bedwiz), dann aber ist das $u$ als $n$ verlesen und so entstand Scealdna im Langfeðgatal, das seinerseits wol wider den ausgangspunkt für Skialldun und Skialldin in SE. und Fl. 1 abgegeben hat.

HALLE a./S., 8. december 1890.

E. SIEVERS.

\section{SINTARFIZILO.}

Gegenuber Mullenhoffs deutung des namens Sintarfizilo Zs. fda. 23, $163 \mathrm{f}$. ist $\mathrm{Kögel}$ in Pauls Grundriss $2^{\mathrm{a}}, 185$ wie ich glaube mit recht zu der ältel'en auffassung J. Grimms zurtuckgekehrt, welcher (Zs. fda. 1,4) in -fizilo ein adj. fizil sah. Ein solches adj. ist als simplex bisher nur einmal in den Pariser Vergilglossen Zs. fda. 15, $42=$ Ahd. gl. 2, 709, 5 belegt, und Mullenhoff meinte daber, dass das fix zelaz dieser stelle leicht fur fizziluehaz verschrieben sein könnte:. Es scheint aber noch ein altes und unanfechtbares zeugnis fur das wort an einer bisher ubersehenen stelle, in des Paulus Diaconus Langobardengeschichte 1,24 vorzuliegen.

Als Alboin um sich die waffen zu lholen zu dem Gepidenkönig Turisind gekommen war, dessem sohn Turismod er im kampfe getötet hatte, und beim mahlee auf Turismods platze 